\title{
The electronic nonadiabatic coupling term: Can it be ignored in dynamic calculations?
}

\author{
G. J. Halász \\ Department of Information Technology, University of Debrecen, P.O. Box 12, H-4010 Debrecen, Hungary
}

\author{
A. Vibók \\ Department of Theoretical Physics, University of Debrecen, P.O. Box 5, H-4010 Debrecen, Hungary
}

S. Suhai

Molecular Biophysics Department, German Cancer Research Center, Im Neuenheimer Feld 280, D-69120 Heidelberg, Germany

M. Baer ${ }^{\text {a) }}$

The Fritz Haber Research Center for Molecular Dynamics, The Hebrew University of Jerusalem, Jerusalem 91904, Israel

(Received 14 August 2007; accepted 12 October 2007; published online 27 December 2007)

\begin{abstract}
Whereas the search for the degeneracy points which are better known as conical intersections (or ci-points) is usually carried out with a lot of devotion, the nonadiabatic coupling terms (NACTs) which together with the adiabatic potential energy surfaces appear in the nuclear Born-Oppenheimer-Schrödinger equation are ignored in most dynamical calculations. In the present article we consider two well known frameworks, namely, the semiclassical surface hopping method and the vibrational coupling model Hamiltonian that avoid the NACTs and examine to what extent, this procedure is justified. (C) 2007 American Institute of Physics. [DOI: 10.1063/1.2806167]
\end{abstract}

\section{INTRODUCTION}

The dynamics triggered in a molecule after a photon is absorbed is usually discussed in terms of the BornOppenheimer (BO) theory ${ }^{1}$ where the fast electronic degrees of freedom are treated separately from those of the slow nuclei. In this picture, electrons and nuclei do not easily exchange energy. Yet, at some nuclear regions, in particular, in the vicinity of degeneracy points this energy exchange is more likely to occur than in other regions. It is widely recognized nowadays that were it not for these degeneracy points important photobiochemical processes such as vision ${ }^{2}$ and photosynthesis of vitamin D could not take place. ${ }^{3}$ It is now accepted that degeneracy points affect also important processes such as photosynthesis in plants ${ }^{4}$ and photochemistry of DNA. ${ }^{5}$

Whereas the search for the degeneracy points which are better known as conical intersections (or ci points) is always carried out with a lot of commitment and devotion ${ }^{6-24}$ (numerous numerical methods are available for this purpose) the nonadiabatic coupling terms (NACTs) which have their origin in the BO theory are hardly ever considered. ${ }^{25}$ It is well accepted that the NACTs are needed to carry out accurate dynamical calculations (transition probabilities, cross sections, rate constants, etc.) However, in general they are either avoided or replaced by other magnitudes. They are ignored when employing the semiclassical surface hopping method $^{26,27(\text { a) }}$ (SSHM) or, also, when applying classical path methods known as Ehrenfest dynamics. ${ }^{27(b), 28}$ They are also

\footnotetext{
a) Author to whom correspondence should be addressed. Electronic mail: michaelb@fh.huji.ac.il
}

ignored when using the so called vibronic-coupling model (VCM) Hamiltonian which is based on parametrized diabatic potential matrix elements that are determined in such a way that the corresponding adiabatic potentials fit $a b$ initio calculations. $^{29}$

There are other, more direct, approaches where the NACTs are imitated by employing alternative magnitudes as, for instance, dipole (or higher orders of) moments ${ }^{30}$ or by considering the overlap between wave functions at neighboring intervals ${ }^{31,32}$ but these will not be discussed here.

In the present article we intend to examine, to some extent, the relevance of the SSHM and the VCM, namely, those approaches that ignore the NACTs. This we do by studying the relationship between the adiabatic hypersurfaces and the corresponding NACTs.

The main assumption in applying the SSHM is that the region which contains the minimum energy path (MEP) is the one preferred by the wave packet while moving from the initial $(j+1, j)$ ci point (to be defined as inlet) to the final $(j, j-1)$ ci point (defined as outlet). ${ }^{33-35}$ Because of that the MEP region is expected to be the region that contributes most to the $j \rightarrow j-1$ electronic transitions. This SSHM assumption is a necessary condition for the importance of the MEP region but there is another condition, not less important, which requires that the spatial distribution of the $(j, j$ -1) NACT overlaps effectively with that MEP region. This aspect which to our knowledge has, so far, not been discussed is a subject in the present article (see Sec. II).

The basic assumption in the VCM is that the adiabatic hypersurfaces and the NACTs are closely linked to each other. In other words, once the adiabatic hypersurfaces $E_{j}(s)$ and $E_{j-1}(s)$ are given the spatial distribution of the relevant 
NACT, $\tau_{j j-1}(s)$ (see below), is also more or less determined. In order to examine the relevance of this assumption we have to derive an analytic expression that connects the NACTs and the adiabatic hypersurfaces. Such an expression is suggested (see Sec. III) and then analyzed numerically (see Sec. V).

The study is carried out for acetylene-a tetra-atomic system considered by us some time ago. ${ }^{36}$ In order to simplify the numerical study we limit ourselves to the $C_{s}$ symmetry.

\section{ON THE SPATIAL OVERLAP BETWEEN NACTS AND HYPERSURFACES}

\section{A. General comments}

In general, a tetra-atomic system is described in terms of six internal coordinates. However, as mentioned earlier, our present study is limited to the $C_{s}$ symmetry where the four atoms are forced to stay on a plane and therefore the positions of the four atoms are described in terms of five coordinates. Next, to make the study both more comprehensive and, mainly, manageable we break up the five dimensional configuration space ${ }^{36}$ and present it as a series of two dimensional configurations. To form the two dimensional configurations we freeze the positions of three atoms, namely, two carbon atoms and one of the hydrogen atoms. The positions of the three atoms are characterized by $R_{\mathrm{CC}}$-the distance between the two carbon atoms, by $R_{\mathrm{CH}}$ - the distance between one of the carbon atoms and the frozen hydrogen, and by $\beta$-the angle formed by $R_{\mathrm{CC}}$ and $R_{\mathrm{CH}}$ (thus $\beta$ $=\angle\left(R_{\mathrm{CC}}, R_{\mathrm{CH}}\right)$, also $\left.\angle(\mathrm{HCC})\right)$. This situation allows the second hydrogen to move freely in the plane formed by the other atoms (see Fig. 1). As is usually the case, this free moving hydrogen is used as a test particle to locate degeneracy or ci points (in Fig. 1, $R$ stands for the distance of one of the ci points from one of the fixed carbon atoms) and it also yields, while moving along a planar contour the spatial distribution of the corresponding NACTs. $\tau_{j k}(\mathbf{s})$ (see below).

\section{B. Electronic nonadiabatic interaction}

In the present article, we consider the four lower states related to the $C_{s}$ symmetry but the numerical study is carried out for two adjacent adiabatic states at a time, namely, the $(j, j-1)$ states; $j=2,3,4$. For this purpose we consider the corresponding NACTs, $\tau_{j j-1}(\mathbf{s})$, defined in general as ${ }^{37}$

$$
\tau_{j j-1}(\mathbf{s})=\left\langle\zeta_{j}\left(\mathbf{s}_{e} \mid \mathbf{s}\right) \mid \nabla \zeta_{j-1}\left(\mathbf{s}_{e} \mid \mathbf{s}\right)\right\rangle
$$

where $s_{e}$ and $s$ stand for a collection of electronic and nuclear coordinates respectively, $\zeta_{i}\left(\mathbf{s}_{e} \mid \mathbf{s}\right) ; i=j, j-1$ are the corresponding adiabatic electronic eigenfunctions and $\nabla$ is the grad operator with regard to the mass-scaled nuclear coordinates.

Having introduced the NACTs we consider the electronic nonadiabatic interaction, $V_{j j-1}(\mathbf{s})$, between the states $j$ and $j-1:^{37}$

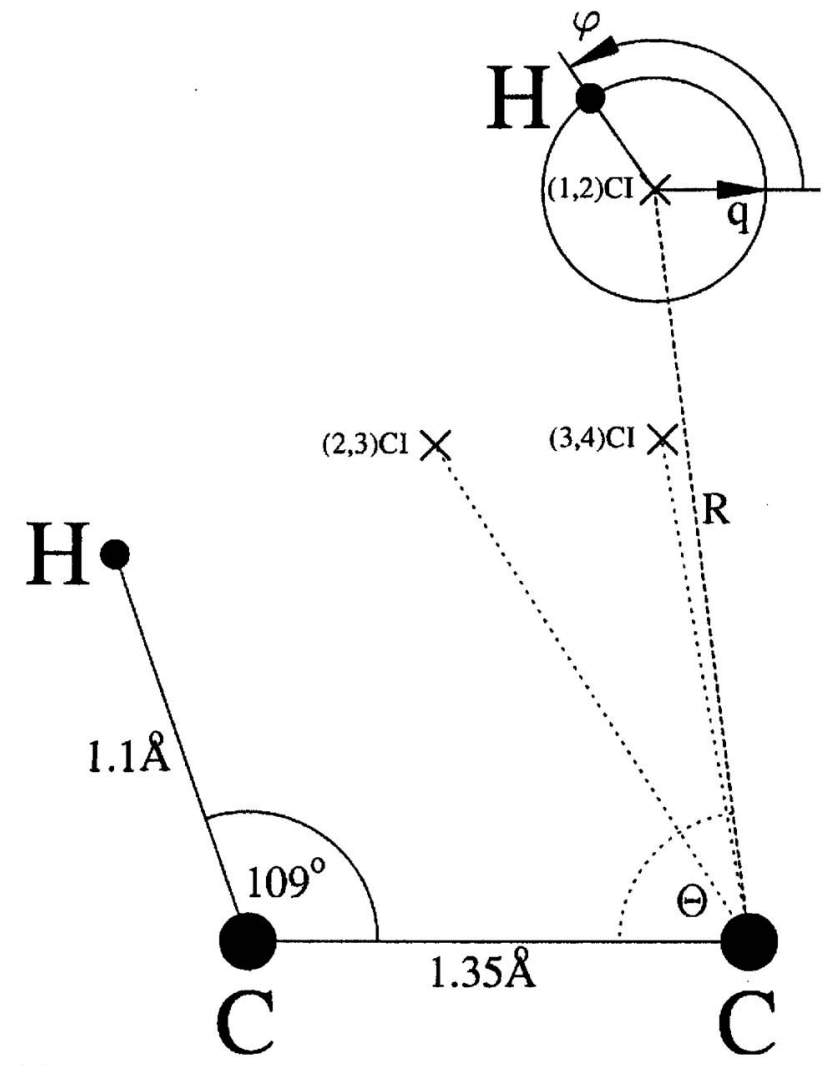

(a)

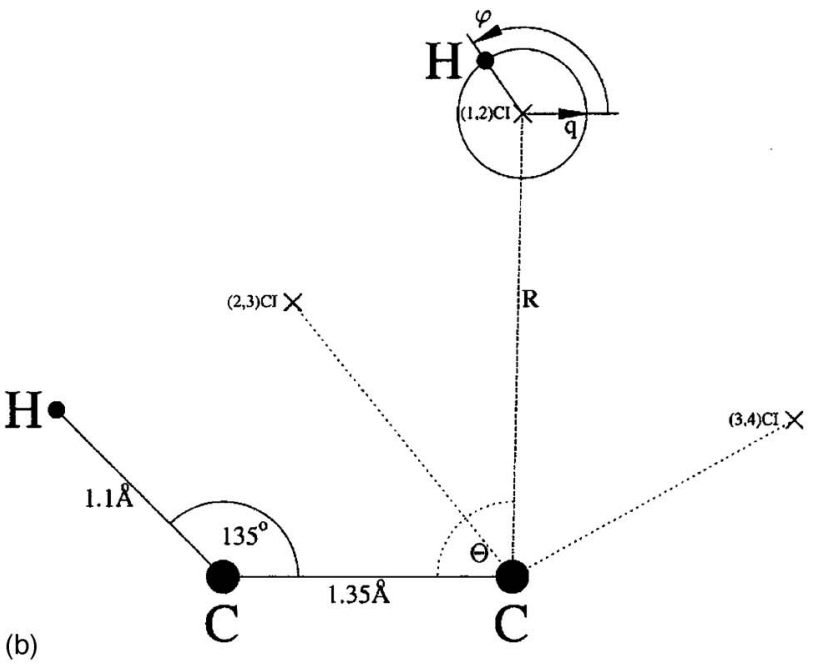

FIG. 1. The $\mathrm{C}_{2} \mathrm{H}_{2}$ configuration and three ci points for which angular NACTs, $\tau_{\varphi j j+1}(\varphi \mid q) ; j=1,2,3$, were calculated. We encounter three fixed atoms, namely, two carbon atoms and one hydrogen. The fourth hydrogen is moving on a fixed plane that contains the four atoms. (a) Results for $R_{\mathrm{CC}}$ $=1.35 \AA, R_{\mathrm{CH}}=1.10 \AA$, and $\beta(\equiv \angle(\mathrm{HCC}))=109^{\circ}$. The positions of the $(j, j+1)$ degeneracy points with respect to the second carbon are as follows $(X$ stands for the free moving hydrogen $):\left(R_{\mathrm{XC}}, \theta_{\mathrm{CCX}}\right)$ $=\left\{\left(2.04 \AA, 82.7^{\circ}\right),\left(1.58 \AA, 57.5^{\circ}\right),\left(1.37 \AA, 80.0^{\circ}\right)\right\}$. (b) The same as in (a) but for $\quad \beta(\equiv \angle(\mathrm{HCC}))=135^{\circ}:\left(R_{\mathrm{XC}}, \theta_{\mathrm{CCX}}\right)=\left\{\left(2.158 \AA, 91.2^{\circ}\right)\right.$, $\left.\left(1.64 \AA, 51.2^{\circ}\right),\left(1.50 \AA, 151^{\circ}\right)\right\}$..

$$
V_{j j-1}(\mathbf{s})=\tau_{j j-1}(\mathbf{s}) \cdot \nabla \psi_{j}(\mathbf{s}) .
$$

This term controls also the intensity of the $j \rightarrow j-1$ transition and it is well noticed that the more effective the overlap between $\tau_{j j-1}(\mathbf{s})$ and the $j$ th component of nuclear wave function, $\psi_{j}(\mathbf{s})$ in a given region, the larger is the contribu- 

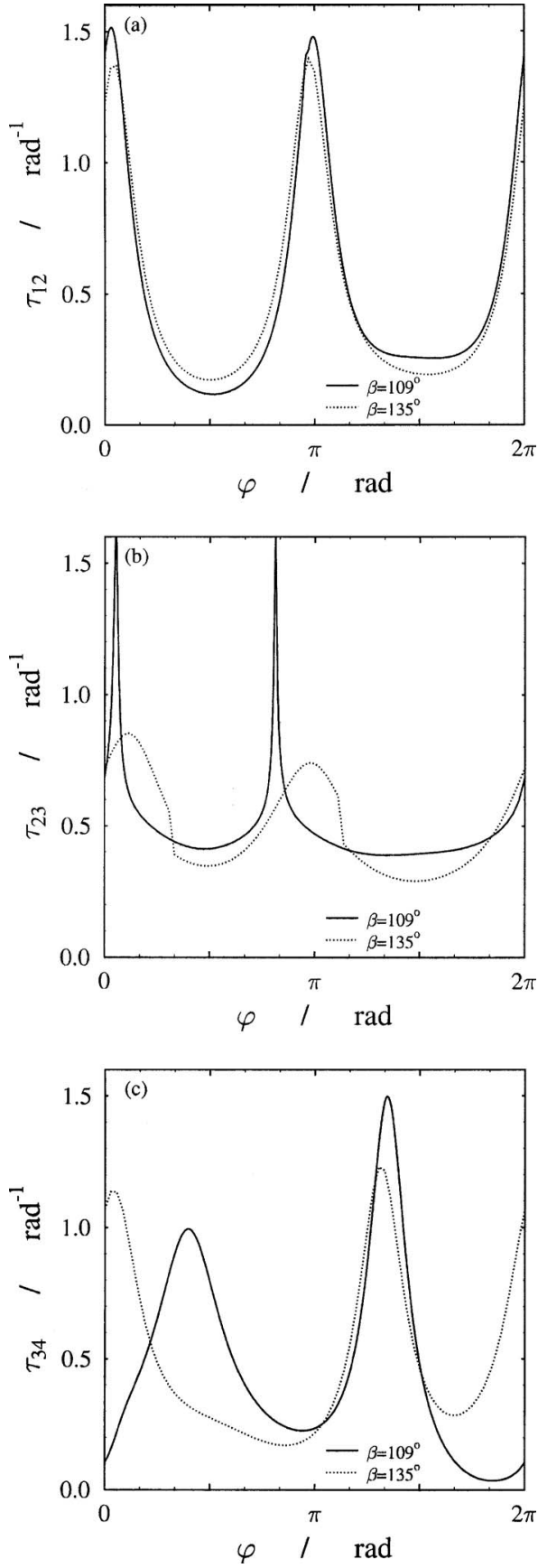

FIG. 2. The angular $\varphi$-dependent NACTs, $\tau_{\varphi j j+1}(\varphi \mid q) ; j=1,2,3$ as calculated for the previously mentioned two configurations along circles, with $q=0.1 \AA$, that surround the various degeneracy points presented in Fig. 1 . The $\tau_{\varphi 12}(\varphi \mid q), \tau_{\varphi 23}(\varphi \mid q)$, and $\tau_{\varphi 34}(\varphi \mid q)$ are presented in panels (a), (b), and (c), respectively. The relevant topological (Berry) phases are $\alpha_{j i+1}(\beta$ $\left.=109^{\circ}\right)=(.3 .1408,3.135,3.124)$ and $\alpha_{j j+1}\left(\beta=135^{\circ}\right)=(3.139,3.092,3.124)$ where the phases in each case are for $j=1,2,3$, respectively.

tion from that region to the overall transition rate.

To continue we elaborate on $\mathbf{s}$ which is assumed to present the following collection of coordinates: $\mathbf{s}=(\varphi, q, z)$, where $\varphi$ (the angle) and $q$ (the radius) are the coordinates of the free hydrogen in the above mentioned plane and $z$ stands for $\left(R_{\mathrm{CC}}, R_{\mathrm{CH}}, \beta\right)$, the coordinates related to the other three atoms. Of the various possible components of $\tau_{j j-1}(\mathbf{s})$ the ones related to the free hydrogen are the radial component $\tau_{q j k}(\varphi \mid q, z)$ and the angular component $(1 / q) \tau_{\varphi j j-1}(\varphi \mid q, z)$. Consequently, Eq. (2) can be written more explicitly as
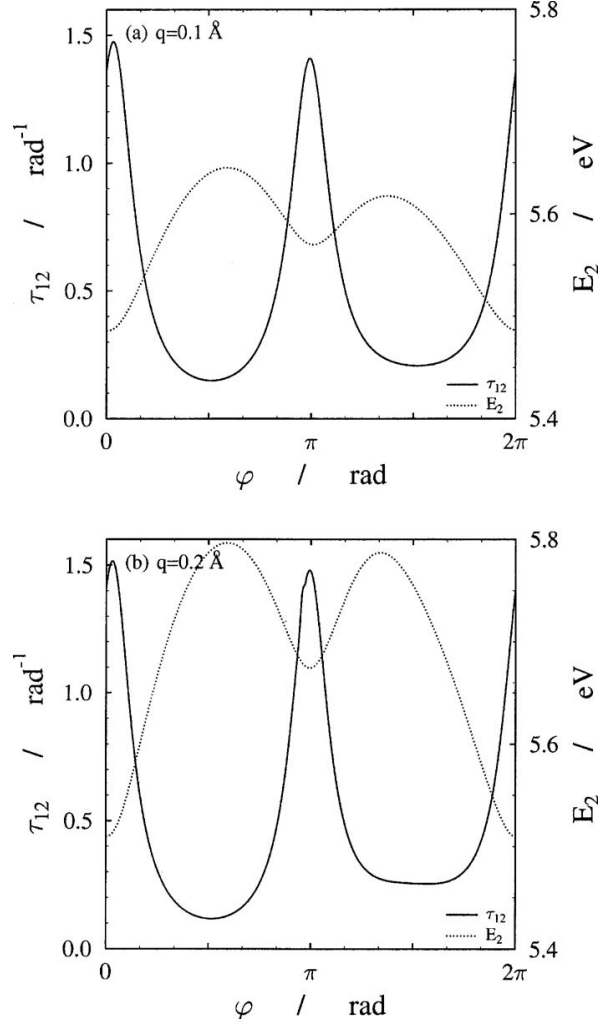

FIG. 3. Representation of the $\varphi$-dependent NACT, $\tau_{\varphi 21}(\varphi \mid q)$ and the $\varphi$-dependent potential hypersurfaces, $E_{2}(\varphi \mid q)$ as calculated for $q=0.1 \AA$ [Fig. 3(a)] and $q=0.2 \AA$ [Fig. 3(b)].

$$
\tau_{j j-1}(\varphi \mid q, z) \cdot \nabla \psi_{j}=\frac{1}{q} \tau_{\varphi j j-1} \nabla_{\varphi} \psi_{j}+\tau_{q j j-1} \nabla_{q} \psi_{j},
$$

where $\nabla_{\varphi}$ and $\nabla_{q}$ are the $\varphi$ and $q$ components of the grad operator, respectively, and the corresponding polar NACTs are known to be

$$
\begin{aligned}
\tau_{\kappa j j-1}(\varphi \mid q, z)= & \left\langle\zeta_{j}\left(\mathbf{s}_{e} \mid \varphi, q, z\right) \mid \frac{\partial}{\partial \kappa} \zeta_{j-1}\left(\mathbf{s}_{e} \mid \varphi, q, z\right)\right\rangle \\
\kappa=q, \varphi . &
\end{aligned}
$$

Next we assume that the two hypersurfaces $E_{j}(\mathbf{s})$ and $E_{j-1}(\mathbf{s})$ form a degeneracy point at $\mathbf{s}=\mathbf{s}_{0}$ and that the origin of the system of coordinates for $(q, \varphi)$ is at $\mathbf{s}_{0}$. Consequently $q \sim\left|\mathbf{s}-\mathbf{s}_{0}\right|$ and therefore as $\mathbf{s} \rightarrow \mathbf{s}_{0}$, the first term in Eq. (3) tends to infinity whereas the second term stays finite. This implies that for any study performed at a relative proximity of the ci, it is enough to consider the angular component of the NACT. Thus recalling Eq. (2) we find that

$$
\lim _{\mathbf{s} \rightarrow \mathbf{s}_{0}} V_{j j-1}(\mathbf{s})=\frac{1}{q} \tau_{\varphi j j-1}(\mathbf{s}) \nabla_{\varphi} \psi_{j}(\mathbf{s}) .
$$

Finally, we return to the SSHM basic assumption, namely, that the wave packet tends to concentrate along the MEP region. Combining this assumption with the $\mathrm{BO}$ interaction term in Eq. (5) it is noticed that in order for $V_{j j-1}(\mathbf{s})$ to yield intense electronic nonadiabatic transitions from the MEP region the overlap between the angular component, $\tau_{\varphi j j-1}$, and the MEP region of $E_{j}(\mathbf{s})$ has to be effective. Our 
numerical study is devoted to this issue and among other things we show that the overlap condition may not always be fulfilled.

\section{A POTENTIAL DEPENDENT MODEL FOR THE NACTS} form $^{38}$

It is well known that the $(j, k)$ NACT is given in the

$$
\tau_{j k}(\varphi \mid q)=\frac{\left\langle\zeta_{j}\left(\mathbf{s}_{e} \mid \varphi, q\right)|\nabla \mathbf{H}| \zeta_{k}\left(\mathbf{s}_{e} \mid \varphi, q\right)\right\rangle}{E_{j}(\varphi, q)-E_{k}(\varphi, q)},
$$

where $\mathbf{H}$ is the electronic Hamiltonian and $E_{i} ; i=j, k$ are the corresponding adiabatic hypersurfaces.

Comment. Here and in what follows, we explicitly mentioned only $(\varphi, q)$ and ignore the $z$ coordinates.

The expression in Eq. (6) shows that $\tau_{j k}$ is proportional to the inverted energy gap $\left\{E_{j}(\varphi, q)-E_{k}(\varphi, q)\right\}^{-1}$ for any $j$ and $k$ and for any component $\tau_{j k}$. Consequently, the analytical expression for the angular NACT, $\tau_{\varphi j j-1}(\varphi \mid q)$, is ${ }^{38(\mathrm{~b})}$

$$
\tau_{\varphi j j-1}(\varphi \mid q)=\frac{\left\langle\zeta_{j}\left(\mathbf{s}_{e} \mid \varphi, q\right)|\partial \mathbf{H} / \partial \varphi| \zeta_{j-1}\left(\mathbf{s}_{e} \mid \varphi, q\right)\right\rangle}{E_{j}(\varphi, q)-E_{j-1}(\varphi, q)}
$$

where the $(1 / q)$ term cancels out on both sides of the equation. Assuming that in the close proximity of the corresponding ci the numerator is approximately independent of $\varphi$ we may replace $\tau_{\varphi j j-1}(\varphi \mid q)$ by its approximate expression $\tilde{\tau}_{\varphi j j-1}(\varphi \mid q)$ defined as

$$
\tilde{\tau}_{\varphi j j-1}(\varphi \mid q)=C_{j j-1}(q)\left\{E_{j}(\varphi, q)-E_{j-1}(\varphi, q)\right\}^{-1},
$$

where $C_{j j-1}(q)$ is a $q$-dependent constant still unknown.

To determine $C_{j j-1}(q)$ we employ a feature which is defined as the quantization of the NACTs. Since we elaborated on this issue on numerous occasions we just briefly refer to it here. We start with the line integral,

$$
\alpha_{j j-1}(\Gamma)=\oint_{\Gamma} d \mathbf{s} \cdot \tau_{j j-1}(\mathbf{s}),
$$

where $\Gamma$ is a closed contour and $\alpha_{j j-1}(\Gamma)$ is frequently referred to the topological (Berry) phase. It is noticed that $\alpha_{j j-1}(\Gamma)$ does not depend on any particular point along the contour $\Gamma$ but only on the contour itself. ${ }^{39}$

Equation (9) simplifies in case the contour is a circle with a radius $q$. For that case $\alpha_{j j-1}(\Gamma)$ becomes $\alpha_{j j-1}(q)$ defined as

$$
\alpha_{j j-1}(q)=\int_{0}^{2 \pi} \tau_{\varphi j j-1}(\varphi \mid q) d \varphi
$$

For an isolated collection of two-state degeneracy points the phase $\alpha_{j j-1}(q)$ [just like $\alpha_{i j-1}(\Gamma)$ ] is expected to be an integer multiple of $\pi$, i.e., $n \pi .^{40,41}$ This fact was shown to exist for numerous realistic systems (see Ref. 25, Sec. IV) and is an essential requirement for diabatic potentials to be single valued (see Ref. 25, Sec. 3.1.1.3).

The fact that for a given $\tau_{\varphi j j-1}(\varphi \mid q)$ the topological phase, $\alpha_{j j-1}(q)$, is quantized is utilized to determine $C_{j j-1}(q)$. Thus substituting Eq. (8) in Eq. (10) and recalling the quantization yield the following equation:

$$
C_{j j-1}(q) \int_{0}^{2 \pi}\left\{E_{j}(\varphi, q)-E_{j-1}(\varphi, q)\right\}^{-1} d \varphi=\pi,
$$

which for every $q$ (and $z$ ) uniquely determines the value of $C_{j j-1}(q)$.

\section{NUMERICAL RESULTS}

Like in previous cases the NACTs (just like the adiabatic hypersurfaces) are calculated at the state-average CASSCF level using 6-311G ${ }^{* *}$ basis set $^{42}$ [employing MOLPRO (Ref. 43)]. We used the active space including all ten valence electrons distributed on ten orbitals (a full valence active space). Following convergence tests, we included in the calculations, in addition to the four studied states, $1^{2} A^{\prime}, 2{ }^{2} A^{\prime}, 3{ }^{2} A^{\prime}$, and $4{ }^{2} A^{\prime}$, also another four to six electronic states of the same symmetry. The positions of the ci points with respect to two different $\mathrm{CCH}$ configurations are presented in Fig. 1. One configuration, namely, $\left(R_{\mathrm{CC}}, R_{\mathrm{CH}}, \beta\right)=(1.35 \AA, 1.10 \AA$, $\left.109^{\circ}\right)$ is identical to the one we considered in Ref. 36 and the second, namely, $\left(R_{\mathrm{CC}}, R_{\mathrm{CH}}, \beta\right)=\left(1.35 \AA, 1.10 \AA, 1.35^{\circ}\right)$ is new (the two configurations differ by the value of the angle $\beta$ ). In the figures we show positions of the available $(1,2)$ and $(2,3)$ cis but not all the $(3,4)$-cis: for the first configuration [Fig. 1(a)] is presented one, out of the two $(3,4)$-cis, ${ }^{36}$ and in the second [Fig. 1(b)] is shown one ci out of three (unpublished) $(3,4)$-cis.

The $\varphi$-dependent NACTs as calculated for the above mentioned two configurations are given in Figs. 2(a)-2(c). In each panel are presented two curves: one is identical to the relevant curve of Ref. 36 and the other as calculated for the new described earlier configuration. All calculations were done for $q=0.1 \AA$. It is noticed that although the NACTs as well as their ci points are sensitive to the assumed configuration (namely, the angle $\beta$ ) still they can be considered as closely related. In the figure captions the various topological phases, $\alpha_{j j+1}(q)$ are listed [see Eq. (10)]. In most cases these phases are close to the expected value of $\pi$.

Since the various features of the just described NACTs are analyzed elsewhere ${ }^{36}$ we continue by referring to the issues as elaborated in the Introduction. In Figs. 3-6 the hypersurfaces $E_{j}(\varphi, q)$ and the corresponding NACTs, $\tau_{\varphi j j-1}(\varphi \mid q)$, as a function of $\varphi$ are presented. We emphasize again that each pair $(j, j-1)$ stands for the $(j j-1)=(2,1)$; $(3,2),(4 a, 3)$ and $(4 b, 3)$-cis [we also remind the reader that the $3 A^{\prime}$ and the $4 A^{\prime}$ states produce two degeneracy points and therefore two NACTs (Ref. 36)]. These results (like other results to be given later) were derived for the first configuration, namely, $\left(R_{\mathrm{CC}}, R_{\mathrm{CH}}, \beta\right)=\left(1.35 \AA, 1.10 \AA, 109^{\circ}\right)$. Each figure is made up of two panels related to two different values of $q$, i.e., $q=0.1,0.2 \AA$ and in each panel two curves are shown: one stands for $E_{j}(\varphi, q)$ and the other for $\tau_{\varphi j j-1}(\varphi \mid q)$.

In Figs. 7-10 the ab initio angular NACTs, $\tau_{\varphi j j-1}(\varphi \mid q)$, with the corresponding approximate potential dependent angular NACTs, $\widetilde{\tau}_{\varphi j j-1}(\varphi \mid q)$ as given in Eq. (8) are compared [see also Eq. (11)]. 

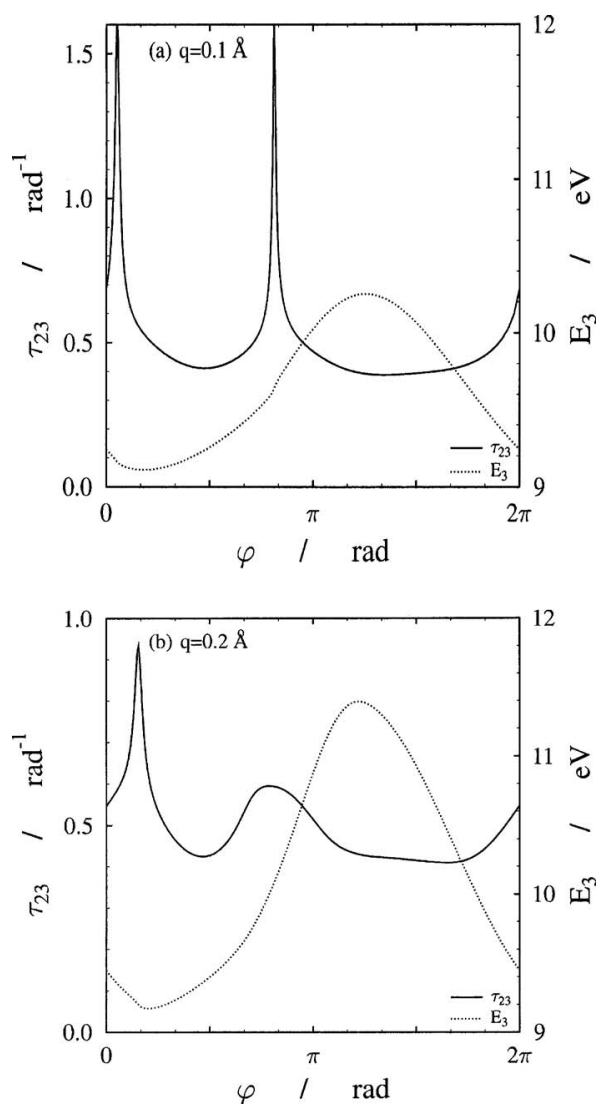

FIG. 4. The same as Fig. 3 but for $\tau_{\varphi 32}(\varphi \mid q)$ and $E_{3}(\varphi \mid q)$.
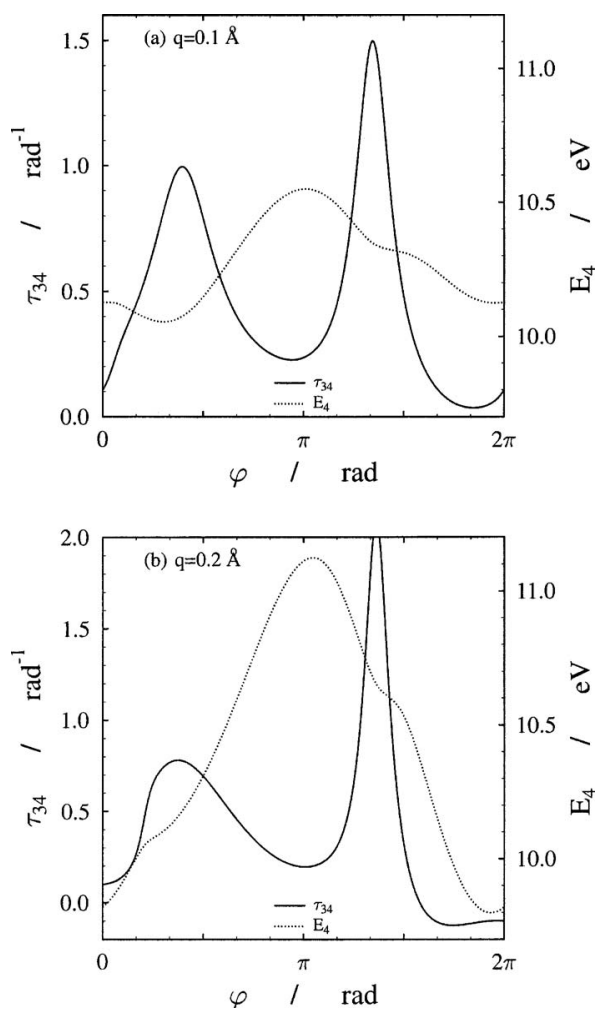

FIG. 5. The same as Fig. 3 but for $\tau_{\varphi 43 a}(\varphi \mid q)$ and $E_{4 a}(\varphi \mid q)$.
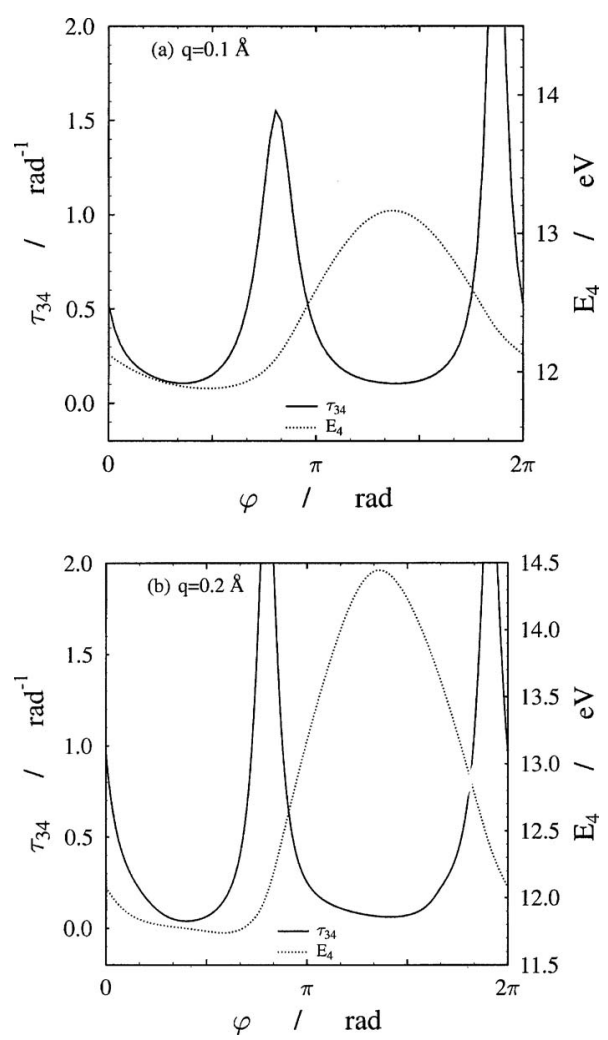

FIG. 6. The same as Fig. 3 but for $\tau_{\varphi 43 b}(\varphi \mid q)$ and $E_{4 b}(\varphi \mid q)$.

\section{ANALYSIS AND CONCLUSIONS}

\section{A. On the overlap between $\tau_{\varphi i j-1}(\varphi \mid q)$ and $E_{j}(\varphi, q)$}

In Fig. $3 \tau_{\varphi 21}(\varphi \mid q)$ with $E_{2}(\varphi \mid q)$ are compared and the comparison shows that there is a reasonable correspondence between the two functions in the following sense: Regions along the MEP where the potentials show a valley (i.e., $\varphi$ $\sim 0, \pi$ ) overlap nicely with intervals where the corresponding NACTs have their maximal values. This phenomenon is evident particularly in the case of $q=0.2 \AA$ [see Fig. 3(b)]. In Fig. $4 \tau_{\varphi 32}(\varphi \mid q)$ and $E_{3}(\varphi \mid q)$ are presented and here the correspondence between the two functions is less visible. In both cases [Figs. 4(a) and 4(b)] the short intervals of the two peaks of $\tau_{\varphi 32}(\varphi \mid q)$ seem to miss the valley along MEP but still the corresponding NACT is nonzero along the region of interest. The situation worsens somewhat when we examine Fig. 5 which shows $\tau_{\varphi 43}(\varphi \mid q)$ and $E_{4}(\varphi \mid q)$ for the lower (lower in energy) ci region, In the case of $q=0.1 \AA$ we find that parts of $\tau_{\varphi 43}(\varphi \mid q)$ (at $\varphi>0$ ) are nonzero along the region of the MEP but for $\varphi<2 \pi(\varphi<0)$ they become close to zero. In the case for $q=0.2 \AA$ it seems that the corresponding NACT is essentially zero along the respective MEP interval. A similar trend is observed in Fig. 6 where we present $\tau_{\varphi 43}(\varphi \mid q)$ and $E_{4}(\varphi \mid q)$ in the close vicinity of the upper ciregion.

The conclusion of this study is that the MEP regions along which we expect, semiclassically, that the wave packet will move from inlet to outlet are not always accompanied with large values of NACTs. Moreover sometimes even the contrary may happen, namely, along portions of the MEP region the relevant NACTs become zero and consequently electronic transitions are almost entirely avoided. 

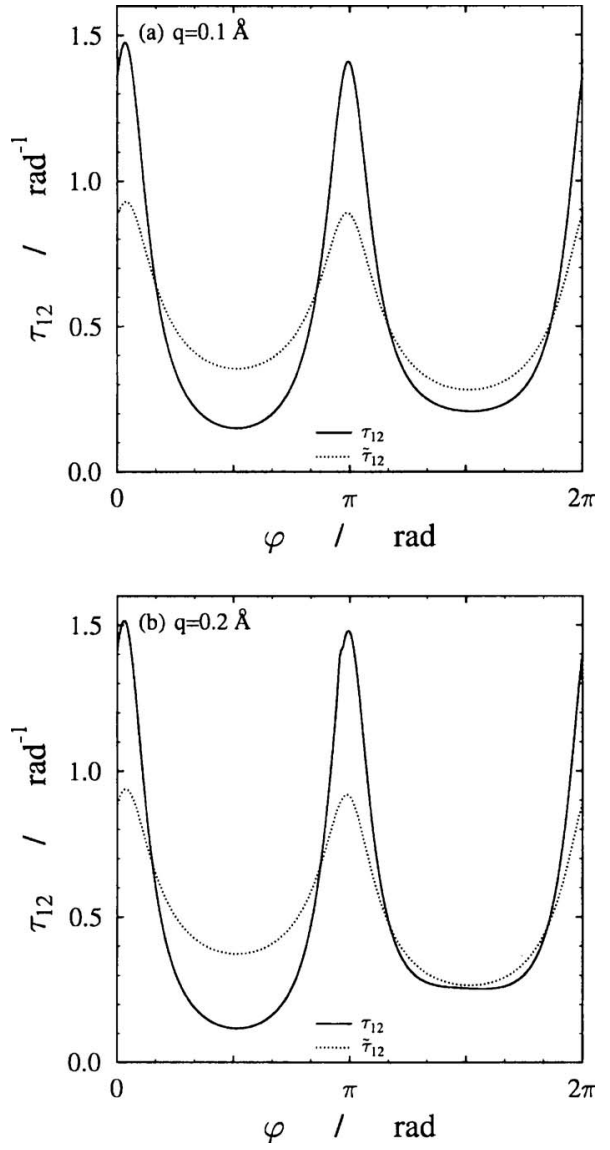

FIG. 7. Comparison between the $\varphi$-dependent ab initio NACT, $\tau_{\varphi 21}(\varphi \mid q)$ and the $\varphi$-dependent model NACT $\widetilde{\tau}_{\varphi 21}(\varphi \mid q)$ as calculated for $q=0.1 \AA$ (a) and $q=0.2 \AA$ (b).

\section{B. On the ability of VCM to yield the NACTs}

In this section we examine to what extent the potential dependent model (introduced in Sec. III) can form reliable angular NACTs which, as we recall, are designated as $\widetilde{\tau}_{\varphi j j-1}(\varphi \mid q)$ [and given by Eq. (8)]. In Fig. 7 the ab initio $\tau_{\varphi 21}(\varphi \mid q)$ function and $\tilde{\tau}_{\varphi 21}(\varphi \mid q)$ for two values of $q$, namely, $q=0.1,0.2 \AA$ are compared. As is noticed both types of NACTs are oscillatory and the fit is reasonable although the $a b$ initio NACTs possess much larger amplitudes. The situation is less encouraging in the case of $\tau_{\varphi 32}(\varphi \mid q)$ and $\tilde{\tau}_{\varphi 32}(\varphi \mid q)$ (see Fig. 8). Here the model NACTs are almost flat whereas the ab initio ones possess strong spikes which could lead to transitions missed by the model. In Fig. 9 (and also in Fig. 10) we find a reasonable fit between the two NACTs in the case of $q=0.1 \AA$ but a less encouraging one for $q=0.2$ for which the $a b$ initio curves (i.e., $\left.\tau_{\varphi 43}(\varphi \mid q)\right)$ are always much more oscillatory than the model ones.

\section{Conclusions}

The feature that characterizes most the angular NACTs is the fact that they are oscillatory (and sometimes even spiky) functions of the (polar) angular coordinate $\varphi$. Since this is a complicated structure it is not easy to reproduce it by models and therefore results acquired by such models cannot always be trusted. For instance, a necessary requirement that the SSHM yield relevant results is that NACTs have suffi-
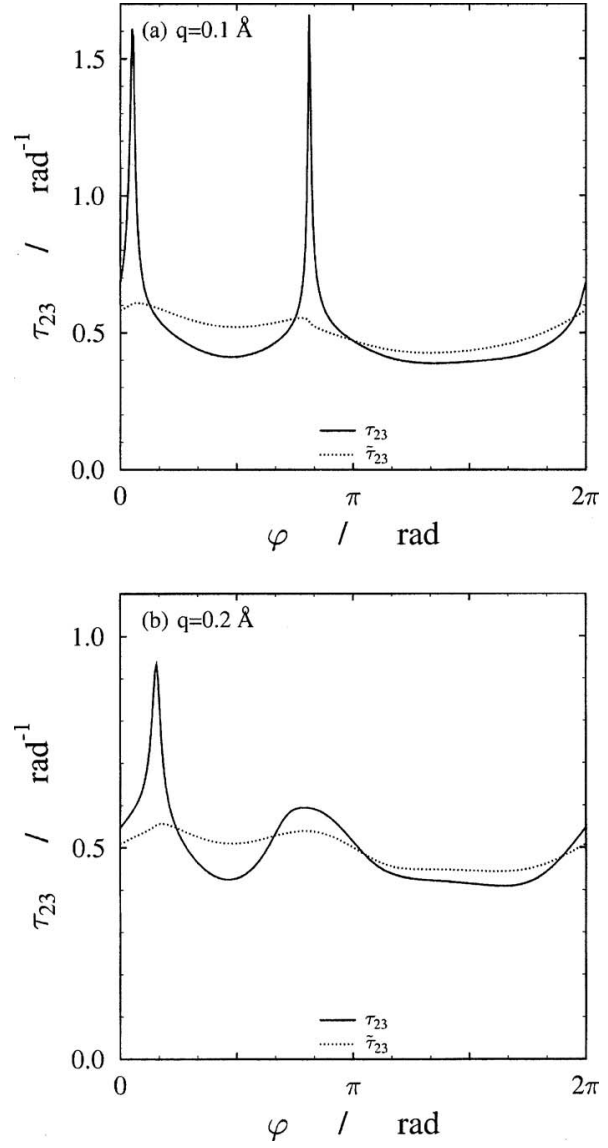

FIG. 8. The same as Fig. 7 but for $\tau_{\varphi 32}(\varphi \mid q)$ and $\widetilde{\tau}_{\varphi 32}(\varphi \mid q)$.

ciently large values in regions that contain the semiclassical MEP (the main region along which the wave packet is moving from inlet-to-outlet). We found that even in our limited study (altogether four cases) this requirement is not always fulfilled, namely, at some instances these NACTs are negligibly small. In this respect, it may be interesting to mention another semiclassical approach, the multiple spawning method which not only follows the motion of the wave packet in the MEP region but also incorporates the effects of NACTs along the classical trajectory. ${ }^{44,45}$ Consequently, this method seems to be more reliable than the other semiclassical approaches (although the details of this approach were not examined by us).

A similar situation is encountered for the VCM. The model is based solely on the knowledge of the adiabatic PESs. Nevertheless the use of this model is justified intuitively, by assuming that the information given by the PESs contains the necessary information to produce the NACTs. To examine this assumption we derived a simple expression for the required (angular) NACTs—see Eq. (8) - based on the adiabatic PESs (and the quantization feature ${ }^{13}$ ).

In this article is argued that justification for using the VCM is that the model-dependent NACTs are sufficiently relevant at least at regions where this model expects the electronic transitions to take place (for instance, along the semiclassical MEP). We find that due to the rather strong oscillatory behavior of the ( $a b$ initio) NACTs the model is frequently inadequate when details are considered. 

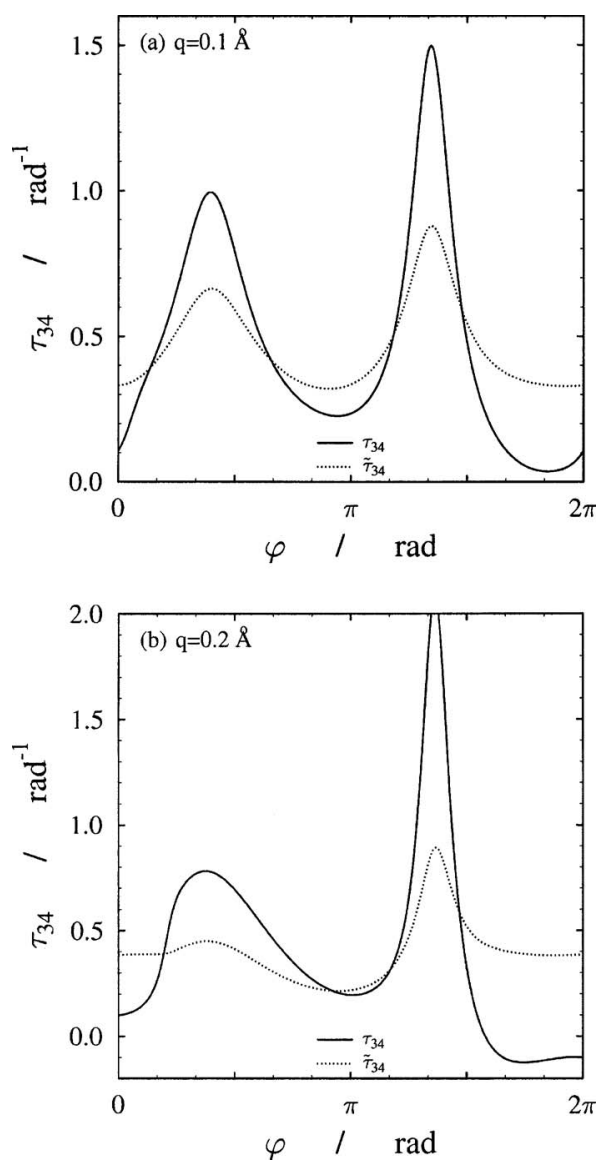

FIG. 9. The same as Fig. 7 but for $\tau_{\varphi 43 a}(\varphi \mid q)$ and $\tilde{\tau}_{\varphi 43}(\varphi \mid q)$.

To our knowledge Eq. (8) was never suggested before (at least not for the purposes as discussed here) and therefore our findings and conclusions can be criticized. However, it seems to us that the expression in Eq. (8) is the simplest way to yield a relation between (angular) NACTs and the adiabatic PESs.

In general both models, the SSHM and the VCM, are believed to be justified. However, it is only after examining the details that we may reveal some weaknesses and sometimes even flaws due to these approaches. If NACTs are becoming negligibly small along the semiclassical MEP then, immaterial how important this region is for storing the wave packet, surface hopping along this region cannot take place. Similar arguments apply to the VCM. It is true that in general the potential dependent model reproduces quite well the $a b$ initio NACTs but it is crucial for them to have a good fit at particular regions as, for instance, along the semiclassical MEP and if they partly fail there the VCM is likely to give inadequate results.

At this stage an important comment has to be made. Our numerical study is based on two-state NACTs which are not affected by other NACTs. If such disturbances become apparent the system is less robust and consequently the required two-state NACTs [as obtained by Eq. (8)] become spikier and less predictable.

\section{ACKNOWLEDGMENTS}

M.B. acknowledges the US-Israel Bi-national Science Foundation for partly supporting this study. A.V. acknowl-
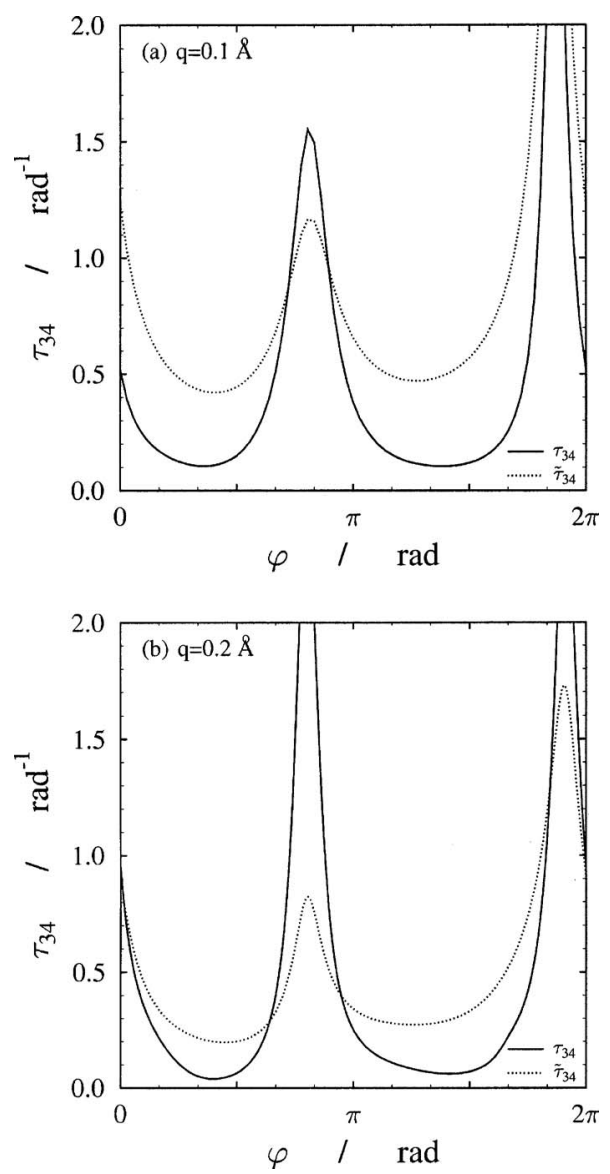

FIG. 10. The same as Fig. 7 but for $\tau_{\varphi 43 b}(\varphi \mid q)$ and $\widetilde{\tau}_{\varphi 43}(\varphi \mid q)$.

edges the OTKA Grant No. T067923 and the computational resources provided by the John-von-Neumann Institute, Research Centre Juelich (Project 1D ehu01). S.S., G.J.H., and A.V. acknowledge the DFG-MTA Bi-national Science Foundation for partly supporting this study.

${ }^{1}$ M. Born and J. R. Oppenheimer, Ann. Phys. 84, 457 (1927); M. Born, Festschrift Göttingen Nach. Math. Phys. K1, 1 (1951); M. Born and K. Huang, Dynamical Theory of Crystal Lattices (Oxford University, New York, 1954), Chap. IV.

${ }^{2}$ P. Kukura, D. W. McCamant, S. Soon, D. W. Wandschneider, and R. A. Mathies, Science 310, 1006 (2005).

${ }^{3}$ F. Bernardi, M. Olivucci, and M. Robb, Isr. J. Chem. 33, 265 (1993)

${ }^{4}$ E. Papagiannakis, J. T. M. Kennis, I. H. M. van Stokkum, R. J. Cogdelle, and R. van Grondelle, Proc. Natl. Acad. Sci. U.S.A. 99, 6017 (2002).

${ }^{5}$ T. Schultz, E. Samoylova, W. Radloff, I. V. Hertel, A. L. Sobolevski, and W. Domcke, Science 306, 1765 (2004).

${ }^{6}$ Conical Intersections: Electronic Structure, Dynamics and Spectroscopy, edited by W. Domcke, D. R. Yarkony, and H. Koppel (World Scientific, Singapore, 2004); in particular, see H. Köppel, ibid. (World Scientific, Singapore, 2004), p. 175; W. Domcke, ibid. (World Scientific, Singapore, 2004), p. 395; S. Mahapatra, ibid. (World Scientific, Singapore, 2004), p. 555; R. de Vivie-Riedle and A. Hofmann, ibid. (World Scientific, Singapore, 2004), p. 829.

${ }^{7}$ M. S. Child and D. E. Manolopoulos, Phys. Rev. Lett. 82, 2223 (1999).

${ }^{8}$ G. Herzberg and H. C. Longuet-Higgins, Discuss. Faraday Soc. 35, 77 (1963); H. C. Longuet Higgins, Proc. R. Soc. London, Ser. A 344, 147 (1975).

${ }^{9}$ E. R. Davidson, J. Am. Chem. Soc. 99, 397 (1977).

${ }^{10}$ R. G. Sadygov and D. R. Yarkony, J. Chem. Phys. 109, 20 (1998).

${ }^{11}$ A. J. Dobbyn and P. J. Knowles, Mol. Phys. 91, 1107 (1997).

${ }^{12}$ R. Xu, M. Baer, and A. J. C. Varandas, J. Chem. Phys. 112, 2746 (2000); A. M. Mebel, M. Baer, V. M. Rozenbaum, and S. H. Lin, Chem. Phys. Lett. 336, 135 (2001). 
${ }^{13}$ M. Baer, S. H. Lin, A. Alijah, S. Adhikari, and G. D. Billing, Phys. Rev. A 62, 032506 (2000); S. Adhikari, G. D. Billing, A. Alijah, S. H. Lin, and M. Baer, Phys. Rev. A 62, 032507 (2000).

${ }^{14}$ T. Takayanagi, Y. Kurosaki, and K. Yokoyama, Chem. Phys. Lett. 321, 106 (2000).

${ }^{15}$ M. S. Child, Adv. Chem. Phys. 124, 1 (2002).

${ }^{16}$ A. Kuppermann, in Dynamics of Molecules and Chemical Reactions, edited by R. E. Wyatt and J. Z. H. Zhang (Dekker, New York, 1996), p. 411; R. Abrol, A. Shaw, and A. Kuppermann, J. Chem. Phys. 116, 1035 (2002)

${ }^{17}$ E. S. Kryachko, Adv. Quantum Chem. 44, 119 (2003).

${ }^{18}$ R. Englman and T. Vertesi, Phys. Lett. A 354, 196 (2006).

${ }^{19}$ M. Baer, T. Vertsi, G. J. Halász, Á. Vibók, and S. Suhai, Faraday Discuss. 127, 337 (2004); A. M. Mebel, G. J. Halász, Á. Vibók, A. Alijah, and M. Baer, J. Chem. Phys. 117, 991 (2002).

${ }^{20}$ P. Barragan, L. F. Errea, A. Macias, L. Mendez, A. Riera, J. M. Lucas, and A. Aguilar, J. Chem. Phys. 121, 11629 (2004); P. Puzari, B. Sarkar, and S. Adhikari, ibid. 121, 707 (2004); B. Sarkar and S. Adhikari, ibid. 124, 074101 (2006).

${ }^{21}$ G. J. Halász, Á. Vibók, A. M. Mebel, and M. Baer, Chem. Phys. Lett. 358, 163 (2002); G. J. Halász, Á. Vibók, A. M. Mebel, and M. Baer, J. Chem. Phys. 118, 3052 (2003); M. Baer, T. Vertsi, G. J. Halász, Á Vibók, and S. Suhai, Faraday Discuss. 127, 337 (2004).

${ }^{22}$ O. Godsi, C. R. Evenhuis, and M. Collins, J. Chem. Phys. 125, 164321 (2006)

${ }^{23}$ V. A. Mozhayskiv, D. Babikov, and A. I. Krylov, J. Chem. Phys. 124, 224309 (2006); K. A. Kistler and S. Matsika, J. Phys. Chem. A 111, 2650 (2007).

${ }^{24}$ E. Abrahamsson, G. C. Groenenboom, and R. V. Krems, J. Chem. Phys. 126, 184309 (2007).

${ }^{25}$ M. Baer, Beyond Born Oppenheimer: Electronic Non-Adiabatic Coupling Terms and Conical Intersections (Wiley, Hoboken, NJ, 2006).

${ }^{26}$ J. C. Tully, Faraday Discuss. 110, 407 (1998); J. C. Tully, in Modern Methods for Multidimensional Dynamics Computations in Chemistry, edited by D. Thompson (World Scientific, Singapore, 1998), p. 34.

${ }^{27}$ G. D. Billing, The Quantum Classical Theory (Oxford University Press, New York, 2003); (a) ibid. (Oxford University Press, New York, 2003),
Sec. 3.3.2; (b) ibid. (Oxford University Press, New York, 2003), Sec. 2.1.3.

${ }^{28}$ G. D. Billing, Int. Rev. Phys. Chem. 13, 309 (1994); P. Puzari, B. Sarkar, and S. Adhikari, J. Chem. Phys. 125, 194316 (2006).

${ }^{29}$ H. Koppel, W. Domcke, and L. S. Cederbaum, Adv. Phys. 57, 59 (1984); S. Mahapatra, G. A. Worth, H.-D. Meyer, L. S. Cederbaum, and H. Koppel, J. Phys. Chem. A 105, 5567 (2001).

${ }^{30}$ A. Mecias and A. Riera, J. Phys. B 11, L489 (1978).

${ }^{31}$ X. Gadea and M. Pellisier, J. Chem. Phys. 93, 545 (1990); T. Romero, A. Aguilar, and X. Gadea, J. Chem. Phys. 110, 6219 (1999).

${ }^{32}$ I. Ryb and R. Baer, J. Chem. Phys. 121, 10370 (2004).

${ }^{33}$ M. Robb, M. Garavelli, M. Olivucci, F. Bernardi, Reviews in Computational Chemistry, edited by K. Lipkowitz and D. Boyd (Wiley, New York, 2000), Vol. 15, p. 87; M. Olivucci, M. Robb, and F. Bernardi, in Computational Analysis of Molecules in Excited State, edited by J. Waluk (Wiley-VCH, New York, 2000), p. 297.

${ }^{34}$ G. A. Worth and M. Robb, Adv. Phys. 124, 355 (2002).

${ }^{35}$ D. R. Yarkony, Faraday Discuss. 127, 325 (2004).

${ }^{36}$ G. J. Halász, Á. Vibók, and M. Baer, Chem. Phys. Lett. 413, 226 (2005).

${ }^{37}$ M. Baer, Chem. Phys. Lett. 35, 112 (1975); Beyond Born Oppenheimer: Electronic Non-Adiabatic Coupling Terms and Conical Intersections (Ref. 25), Sec. 2.1.1.

${ }^{38}$ (a) S. T. Epstein, Am. J. Phys. 22, 613 (1954); (b) Beyond Born Oppenheimer: Electronic Non-Adiabatic Coupling Terms and Conical Intersections (Ref. 25), Sec. 5.1.

${ }^{39}$ Beyond Born Oppenheimer: Electronic Non-Adiabatic Coupling Terms and Conical Intersections (Ref. 25), Sec. 3.1.

${ }^{40}$ T. Vertesi, A. Vibok, G. J. Halasz, and M. Baer, J. Chem. Phys. 120, 2565 (2004).

${ }^{41}$ D. Bohm, Quantum Theory (Dover, New York, 1989), p. 41.

${ }^{42}$ R. Krishnan, M. Frisch, and J. A. Pople, J. Chem. Phys. 72, 4244 (1980).

${ }^{43}$ MOLPRO, a package of ab initio programs written by H.-J. Werner and $\mathrm{P}$. J. Knowles with contributions from J. Almlöf et al.

${ }^{44}$ T. J. Martinez, M. Ben-Nun, and R. D. Levine, J. Chem. Phys. 100, 7884 (1996); J. Phys. Chem. A 101, 6389 (1997).

${ }^{45}$ A. Toniolo, S. Olsen, L. Manohar, and T. J. Martinez, Faraday Discuss. 127, 149 (2004). 